\title{
La douleur en 3D : Dent, Dentiste, Douleur
}

\author{
Sous la présidence scientifique du Dr Luc Chikhani, \\ à la Fondation de la Maison de la chasse et de la nature, \\ Hôtel de Guénégaud, 60-62, rue des Archives, 75003 Paris
}

\section{SÉANCE DU JEUDI 11 OCTOBRE 2012}

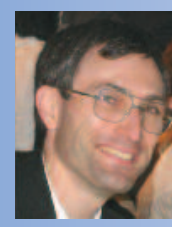

Christophe BONNEFOY

78 , avenue Aristide Briand 92160 Antony.
Cette séance, organisée de main de maître par notre confrère le Dr Luc Chikhani, président scientifique de notre Association d'Enseignement d'Odontologie et de Stomatologie, traitait, sur la base de six courtes conférences, de la délicate question de la douleur en odontostomatologie avec un titre digne d'un blockbuster hollywoodien : "La douleur en 3D : Dent, Dentiste, Douleur ». Elle s'est tenue dans le cadre rituel, accueillant et toujours aussi propice à la réflexion et à la concentration, de l'amphithéâtre de I'Hôtel de Guénégaud. En dernière partie, toujours dans la tradition de l'AEOS, le débat s'est élargi sur le plan médical à une autre spécialité en traitant du syndrome du canal carpien.

Des conférences courtes et précises sur des sujets importants pour la pratique, voilà manifestement les consignes qu'avaient reçues nos conférenciers et qu'ils ont bien respectées, suscitant dans une assistance assez fournie un nombre impressionnant de questions très intéressantes, preuve s'il en était besoin, que cette formule fonctionne toujours aussi bien.

Notons que cette séance aurait aussi pu s'intituler « Tout ce que vous auriez aimé qu'on vous apprenne sur la douleur " pendant vos chères études afin de vous sortir du pétrin dans lequel celle-ci vous a mis en pratique. À une époque où le numérique, le virtuel et la biologie moléculaire envahissent notre discipline sans réellement toujours apporter leur lot de réponses à ces questions pourtant non dénuées d'intérêt, il est bon de revenir aux fondamentaux. En effet, la douleur est, avec l'aspect financier, le principal acteur dans la perte de patient au cabinet dentaire ainsi que dans la mythique « peur du dentiste ». 


\section{Évolution visuelle analogique de la douleur Dr Frédéric Larché, stomatologiste}

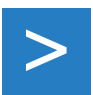

Cette conférence amorcée avec brio par une diapositive d'une toile bien de circonstance, Le cri d'Edward Munch, nous a plongé dans les différentes échelles d'évaluation de la douleur.

On distingue ainsi les échelles multidimensionnelles comme celle basée sur un carnet d'évaluation, ou sur le schéma corporel des zones douloureuses, le Mac Gill Pain Questionary et sa version française que constitue le Questionnaire Saint-Antoine et enfin l'Hospital Anxiety Depression Scale. Ces derniers peuvent surtout concerner dans notre discipline la cancérologie et la chirurgie maxillofaciale.

Les échelles unidimensionnelles sont plus simples, plus rapides à mettre en œuvre et bien adaptées à la douleur dentaire. On distingue : l'échelle verbale simple, l'échelle numérique et l'échelle visuelle analogique notée de 0 à 10 . Cette dernière est reprise dans la classification de I'OMS qui fait référence à trois paliers, correspondant chacun à trois thérapeutiques différentes. Le praticien demande au patient d'évaluer l'intensité de sa douleur en déplaçant un curseur sur cette échelle. Cette méthode reste malheureusement assez subjective en absence d'évaluation réellement scientifique des stimuli nociceptifs mais elle fonctionne cependant assez bien d'une façon générale, d'autant qu'elle permet de distinguer trois paliers :

- le palier 1, qui correspond à une douleur d'intensité modérée, de 1 à 4 , traitée par les antalgiques non-opioïdes comme le paracétamol et les AINS ;

- le palier 2, qui correspond à une douleur d'intensité intermédiaire, de 4 à 7 , traitée par les

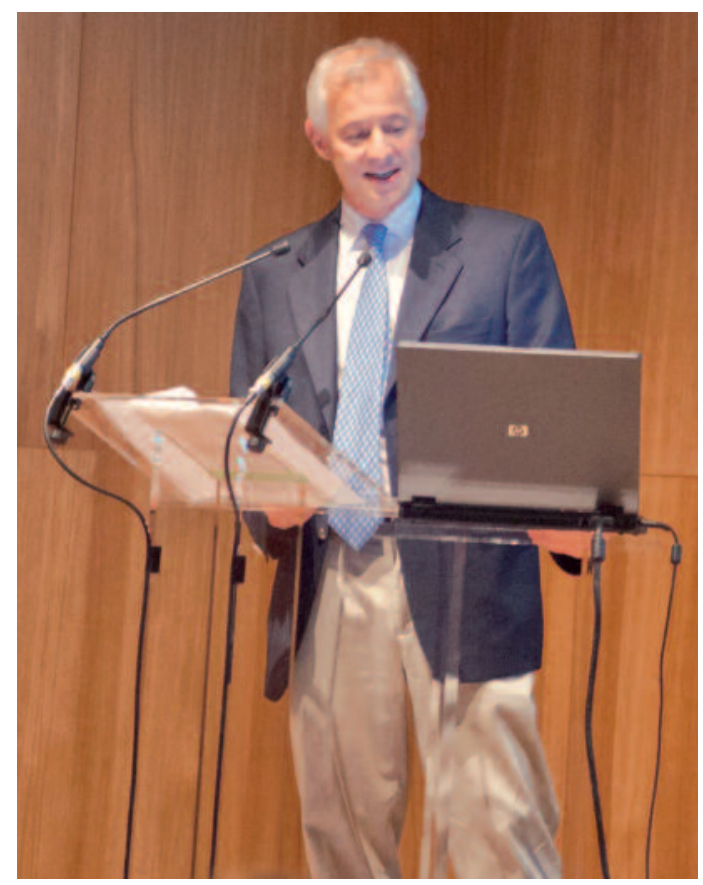

antalgiques opioïdes faibles comme le tramadol avec ou sans association au paracétamol, et le tandem paracétamol-codéine.

- le palier 3, qui correspond à une douleur d'intensité forte, de 7 à 10, traitée pour cette raison par les opioïdes forts qui restent heureusement et fréquemment peu prescrits dans notre spécialité comme la morphine (Skenan $\mathrm{LP}^{\circledR}$ ), le fentanyl transdermique (Durogesic ${ }^{\circledR}$ ) et l'hydromorphone (Sophidone LP ${ }^{\Theta}$ ) dans la catégorie des agonistes purs ou comme les agonistes antagonistes avec la nalbulphine (Nubain ${ }^{\circledR}$ ) et enfin les agonistes partiels comme la buprénorphine (Temgésic ${ }^{\circledR}$ ).

Et le conférencier de conclure par un autre tableau beaucoup moins connu d'Edward Munch, Nuit d'été, mais dont le caractère calme et apaisant convenait bien à la réussite d'une médication bien conduite et raisonnée. 


\section{Alvéolite sèche}

\section{Dr Jean-François Legrand, chirurgien-dentiste}

Voilà la tant redoutée et si injuste pourvoyeuse de sérieux problèmes au cabinet dentaire : la fameuse douleur du troisième jour qui concerne jusqu'à environ $15 \%$ des avulsions des dents mandibulaires. Tant redoutée à cause de l'effritement du moral et de la confiance du patient en son praticien devant des algies perturbant son sommeil et si injuste car elle peut arriver sur n'importe quelle dent, y compris celle n'ayant causé aucune difficulté lors de l'avulsion contrairement aux idées reçues.

Il s'agit en fait d'un défaut de vascularisation de l'alvéole, le caillot ne remplit pas alors son rôle protecteur régénérateur sur I'os sousjacent et se désagrège dans les jours qui suivent l'intervention. L'alvéole apparait alors comme " déshabitée » dans la description qui en est couramment formulée.

Le cas clinique bien classique et brutal est donc l'urgence au quatrième jour, le patient n'ayant pas pu (ou peu) dormir de la nuit, ce qui implique nécessairement une douleur de type 3 sur l'échelle visuelle analogique dont on imagine aisément le retentissement psychologique. Le diagnostic d'alvéolite sèche est posé, en absence d'une suppuration évidente, en face de la tétrade bien connue : douleur, chaleur, rougeur, tuméfaction.

Beaucoup de remèdes ont été proposés dont les mèches iodoformées mais le plus efficace prôné par le conférencier consiste à mécher l'alvéole avec une petite compresse de gaze imprégnée d'huile essentielle de clou de girofle. Le patient est invité à venir au cabinet la remplacer tous les deux jours. En général, la troisième application est suffisante et les antalgiques classiques de palier 1 peuvent prendre le relais jusqu'à la

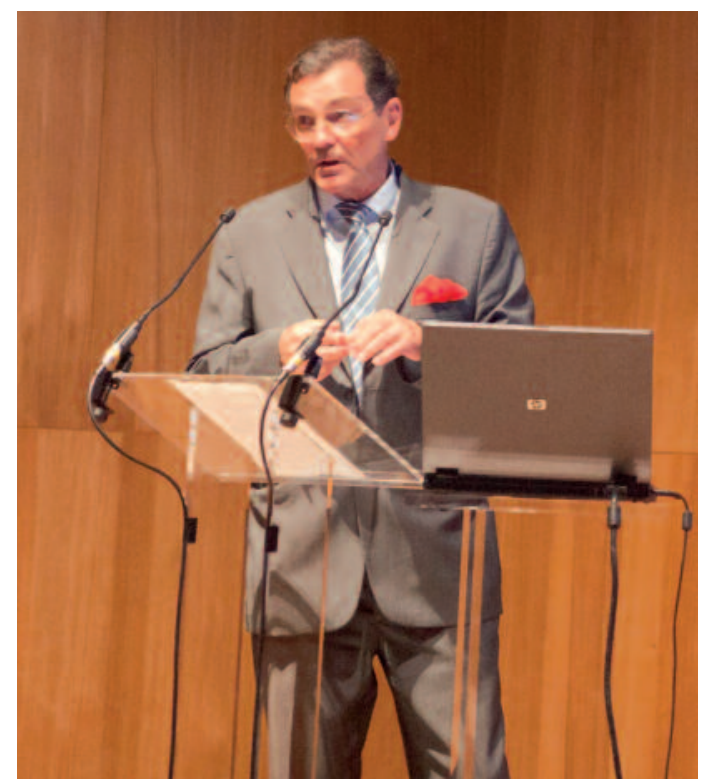

cicatrisation de deuxième intention qui survient au $13^{\mathrm{e}}$ jour.

Ce remède simple, efficace et peu onéreux, permet de retrouver la confiance du patient atteint et une tranquillité d'esprit nécessaire à la concentration qu'exige le travail au fauteuil.

Beaucoup de moyens ont été proposés pour prévenir l'apparition d'une alvéolite : cureter systématiquement l'alvéole après avulsion, ne pas échauffer l'os pendant le fraisage, ne pas utiliser de vasoconstricteurs... Force est de constater que; malgré toutes ces précautions, des cas d'alvéolites sont retrouvés à la fois dans la littérature et la clinique.

Il a été rapporté que les changements de pressions barométriques favorisaient les alvéolites mais le caractère aléatoire des facteurs météorologiques ne permet pas d'en faire une prévision satisfaisante pour fixer les jours d'interventions les plus propices au bon déroulement des suites post-opératoires. 


\section{Gestion de la douleur dentaire en pratique quotidienne} Dr Valérie Travert, chirurgien-dentiste

Les douleurs dentaires sont les principaux motifs de consultation au cabinet dentaire pour lesquels le praticien doit poser un diagnostic et entreprendre, quand cela est possible, un acte thérapeutique qui soulage efficacement le patient, sans oublier la place de la prescription médicamenteuse qui est indispensable dans certaines situations cliniques.

La douleur diffère en nature et en intensité, et ses caractères peuvent suffire à établir le diagnostic de l'affection causale, ou orienter l'examen avec précision. On gère alors le cas selon la sévérité de la pathologie dentinaire, pulpaire ou péri-apicale pouvant aller jusqu'à la cellulite aigue circonscrite.

S'il est bien connu qu'il suffit de couvrir une mise à nue dentinaire pour en apaiser les symptômes, il n'en va pas de même de la pulpite qui reste un motif de consultation fréquent et potentiellement problématique au cabinet dentaire. En urgence, on ne peut souvent se contenter que d'une pulpotomie mais il est admis qu'un traitement endodontique mené en une seule séance est un gage de succès thérapeutique et symptomatique. Les AINS sont intéressants pour calmer la desmodontite réactionnelle à la thérapeutique endodontique mais ils ne doivent pas être utilisés en cas de risque d'extension de la pathologie aux tissus avoisinants. D'une façon générale, si la douleur persiste, on prescrit du paracétamol ou une association paracétamol-codéine si elle reste importante.

La pathologie péri-apicale doit être combattue par un drainage des exsudats péri-apicaux

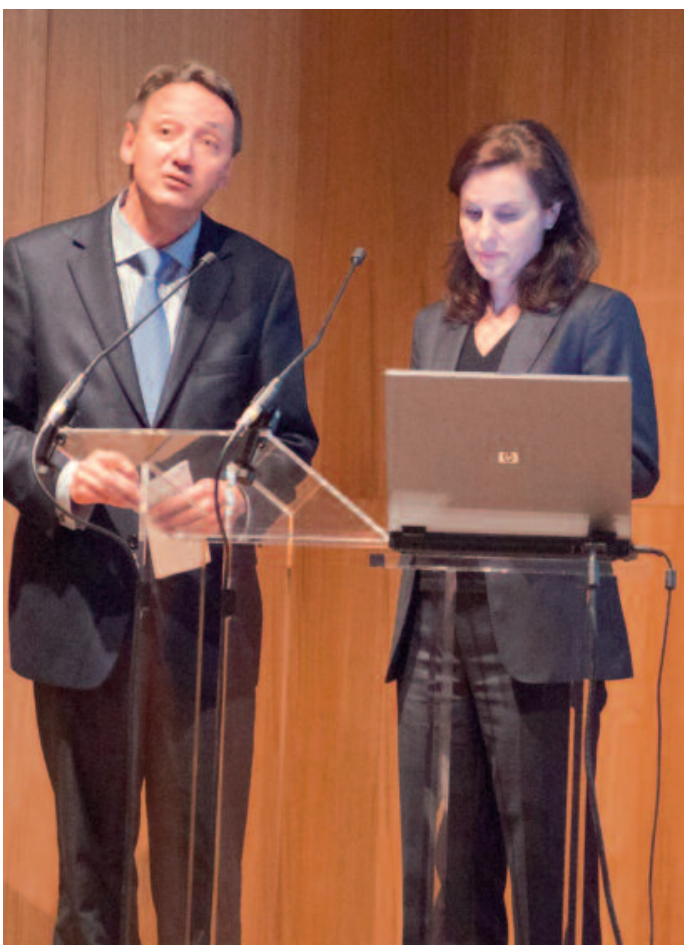

et une désinfection concomitante de façon à réduire non seulement la pression desmodontale mais aussi la charge bactérienne. Si le drainage est effectué, les antibiotiques, contrairement à une idée reçue, ne sont pas nécessaires à l'exception d'une altération de l'état général. On prescrira alors I'amoxicilline ( $2 \mathrm{~g} / \mathrm{j}$ pendant 5 jours) ou la pristinamycine $(2$ à $3 \mathrm{~g} / \mathrm{j})$ ou la clindamycine $(1,2$ à $2,4 \mathrm{~g} / \mathrm{j}$ pendant 5 jours).

Si le drainage est impossible, on laisse la dent ouverte pendant 2 jours avec l'association : amoxicilline 1,5 g/j + métronidazole $0,75 \mathrm{~g} / \mathrm{j}$ pendant 5 jours.

Les antalgiques de palier I ou II sont généralement efficaces d'autant que la guérison de 
I'infection par la thérapeutique antibiotique diminuera l'inflammation et par conséquent la pression et les douleurs qui en résultent. C'est pourquoi les AINS ne sont pas nécessaires et même contre-indiqués à cause du risque d'extension de l'infection surtout en l'absence d'antibiotiques.

Enfin en ce qui concerne la cellulite aiguë circonscrite, le drainage est impératif et l'anti- biothérapie systématique pour éviter le risque de diffusion systémique de l'infection, I'antibiotique devant accompagner et non pas remplacer le geste chirurgical afin d'éviter un passage à la chronicité.

Le praticien peut ainsi, à partir du diagnostic, maîtriser son geste thérapeutique et prescrire les bonnes médications de façon à agir « au bon endroit, au bon moment $"$.

\section{Douleur en pathologie salivaire Dr Agnès Guerre, chirurgien maxillofacial}

Les glandes salivaires sont dissociées classiquement en glandes salivaires principales comme la parotide, la submandibulaire et la sublinguale, paires et symétriques, et en glandes salivaires accessoires qui tapissent la cavité buccale. Deux types de pathologies sont représentés : la pathologie tumorale et la pathologie non tumorale. Le second type est le plus fréquent puisqu'il s'agit d'une sorte de fourre-tout où l'on trouve :

- des pathologies rétentionnelles (lithiasique ou sténosante) ;

- des pathologies infectieuses (post-rétentionnelle ou primitive) ;

- des maladies de système et troubles du comportement alimentaire ;

- des pathologies iatrogènes (iode 131, radiothérapie) ;

- des psychopathologies et médicaments sialoprives.

Le premier type est également riche puisqu'il contient les tumeurs bénignes - dont la plus commune est l'adénome pléomorphe - et malignes. À cela viennent s'ajouter les patho-

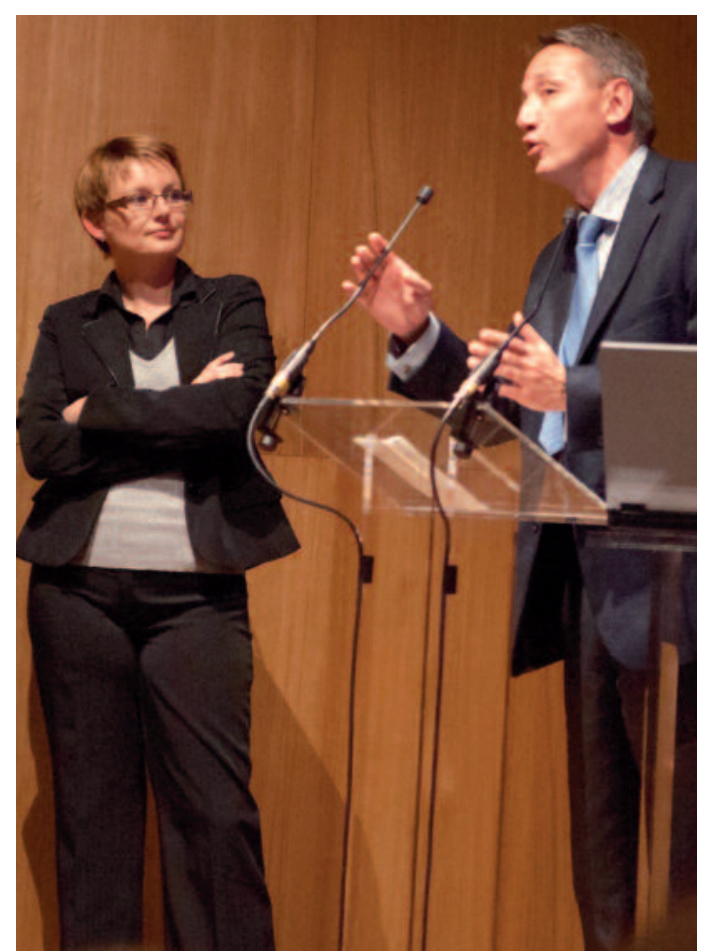

logies kystiques (kystes mucoïdes, kystes salivaires, sialocèles...) et nodulaires. Dans le lot, il convient de distinguer les pathologies à fort potentiel nociceptif de celles qui ne le sont pas et qui peuvent n'en être que plus insidieuses. La conférencière a dénombré un premier 
groupe d'environ sept pathologies douloureuses contre un second groupe de quatre pour les pathologies qui ne sont pas censées créer de troubles nociceptifs.

Dans le premier groupe, la colique salivaire tient une place de choix. Il peut s'agir d'une lithiase ou d'une sténose provoquant des épisodes de tuméfaction douloureux lors des repas. La levée de l'obstacle est impérative car sinon le risque de complication infectieuse en sialite serait inévitable. Les parotidites ou submandibulites peuvent donc se produire par stagnation salivaire et infection rétrograde ou être primitives (parotidite bactérienne de l'enfant, parotidite virale surinfectée). On observe alors une tuméfaction avec rougeur, érythème cutané, pus à l'ostium, difficulté à s'alimenter, fièvre et altération de l'état général.

Les maladies de système comme le syndrome de Gougerot-Sjögren peuvent provoquer des tuméfactions par blocage avec atteinte douloureuse du parenchyme, provoquant des sialites et en particulier des parotidites. Les séquelles de radiothérapie cervico-faciale ou métabolique à l'iode 131 provoquent une sclérose des glandes salivaires avec des épisodes douloureux avec ou sans tuméfactions. Et enfin la xérostomie perturbe la qualité de vie des patients qui en sont atteints et on peut dire en ce sens qu'une bouche sèche "fait mal ».

Enfin, par la gravité, le premier groupe est dominé par les tumeurs malignes, qui ne sont cependant pas systématiquement symptoma- tiques, et les nodules infectés ou inflammatoires en région parotidienne. Notons qu'il n'existe pas de nodules en région submandibulaire, ce qui doit faire suspecter d'emblée la présence d'une tumeur.

La conduite à tenir est de toujours traiter l'infection avant de recourir à la sialographie, notamment dans les coliques salivaires, l'échographie permettant de mieux en mieux de préciser l'étiologie, notamment tumorale, en association avec I'IRM.

Au sein du deuxième groupe formé par les pathologies qui ne sont pas symptomatiques, on retrouve les hernies salivaires qui sont des accidents mécaniques rythmés par les repas avec blocage transitoire et incomplet du flux salivaire. Les tumeurs bénignes sont également représentées quoiqu'il puisse exister parfois des tumeurs malignes indolores, éventualité à laquelle il faudra toujours penser. Enfin, les kystes (mucoïdes et les sialocèles) et les hypertrophies parotidiennes par alcool ou dysorexies complètent le vaste éventail de pathologies sans retentissement douloureux que le clinicien est susceptible de rencontrer. La conférencière a réussi à faire passer son message en un court laps de temps sur une pathologie fort complexe et qui n'est pas toujours bien facile à identifier par des non-spécialistes. En effet, le diagnostic différentiel devra toujours être réalisé afin d'éliminer une dysfonction de l'ATM, une hypertrophie massétérine, un syndrome d'Eagle, des nodules dans la loge sous-mandibulaire et des étiologies dentaires variées. 


\section{Douleur en implantologie Dr Marc Bert, chirurgien-dentiste}

" Les implants ne font pas mal sauf quand ils font mal ». C'est par cet aphorisme volontiers provocateur que notre conférencier, le Dr Marc Bert, a présenté la douleur en implantologie.

Effectivement, la douleur n'est pas une composante normale de la symptomatologie postopératoire en implantologie et, à ce titre, elle n'est pas de bon augure pour la pérennité du ou des implants concernés. Elle partage un certain " timing " avec la douleur de l'alvéolite sèche puisqu'elle est également une douleur du troisième jour. Elle aboutit à un plateau à la fin de la première semaine puis régresse seulement au cours de la troisième semaine pour finir par disparaitre à la fin de celle-ci quand il est trop tard, l'implant baignant dans un tissu fibreux sans intérêt mécanique. Les radiographies montrent une hyperpression au niveau de l'apex implantaire qui gagne progressivement mais rapidement toute la périphérie osseuse, allant jusqu'à créer progressivement des paresthésies au voisinage du canal mandibulaire.

Fort de ce constat et afin de perdre le moins d'os possible, il est nécessaire d'éviter tout acharnement thérapeutique en déposant au plus tôt le ou les implants concernés et de recommencer l'implantation quelques semaines plus tard selon un protocole bien défini. Cette conduite possède un autre intérêt qui se révèle crucial pour le patient concerné : la sédation très rapide de la douleur par suppression directe de sa cause, ce qui l'incitera à retrouver une certaine confiance en son praticien pour la réimplantation ultérieure.

Bien sûr, de nombreuses études ont porté sur les circonstances favorisant cette douleur implantaire du troisième jour. II est manifeste que

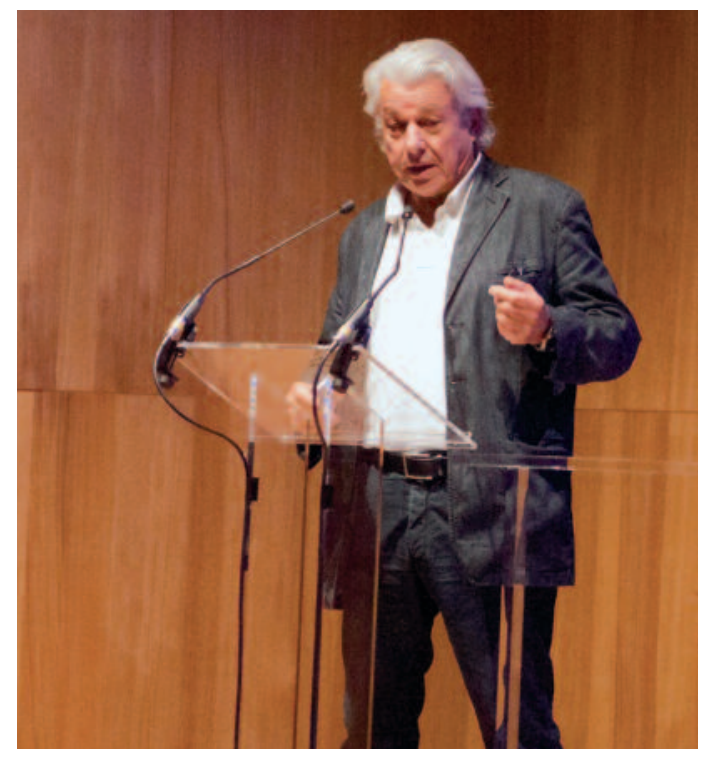

l'échauffement d'un foret, même sous irrigation abondante, peut favoriser la survenue de cette complication qui signe toujours l'échec de l'implant à court ou moyen terme. Mais le vissage avec un couple de serrage trop élevé à 40-45 N comme il est préconisé par certains à la mandibule, en vue de réaliser une implantation immédiate par exemple, accentue le risque en créant une hyperpression sur l'os péri-implantaire.

Se prémunir totalement de ce problème n'est pas réellement possible car, comme aime le rappeler le Dr Marc Bert, l'échec est statistiquement prévisible sur de grandes séries mais non individuellement prédictible. II est donc nécessaire d'expliquer au patient avant toute intervention que quelques cas de ce type peuvent survenir surtout à la mandibule et de le rassurer en lui expliquant que rien n'est perdu afin de prévenir au maximum le lot habituel d'insatisfaction et d'anxiété que suscitera nécessairement l'apparition de cette complication tant redoutée en implantologie. 


\section{Imagerie de la douleur dentaire Dr Gérard Pasquet, radiologue}

La douleur est un motif de consultation essentiel en imagerie dento-faciale. Le cliché panoramique dentaire est l'incontournable examen complémentaire de première intention face à des douleurs diffuses aigues ou chroniques maxillo-mandibulaires ou localisées mandibulaires. Mais cette technique ne permet pas d'imagerie sectionnelle particulièrement en coupes axiales, bien utiles dans certains cas insidieux.

En effet, lors des douleurs localisées aigues ou chroniques, notamment causées par des fêlures ou des fractures radiculaires ou les dépassements de pâtes, le cone beam, ou CBCT scan, se révèle non seulement en tant que technique d'imagerie de confirmation mais surtout également diagnostique lorsque le cliché panoramique ne laisse apparaître aucune pathologie.

On utilise alors le CBCT en petit champ haute résolution à voxels de $0,125 \mathrm{~mm}$ ramenés à $0,08 \mathrm{~mm}$ en rétro-reconstruction. Cet examen se révèle alors efficace en précision sur ces cas problématiques puisque l'imagerie conventionnelle ne suffit pas toujours à les détecter et qu'en conséquence, on ne sait pas toujours quelle conduite adopter.

Cette technique est d'autant plus intéressante que l'on connait son efficacité sur les tissus durs,

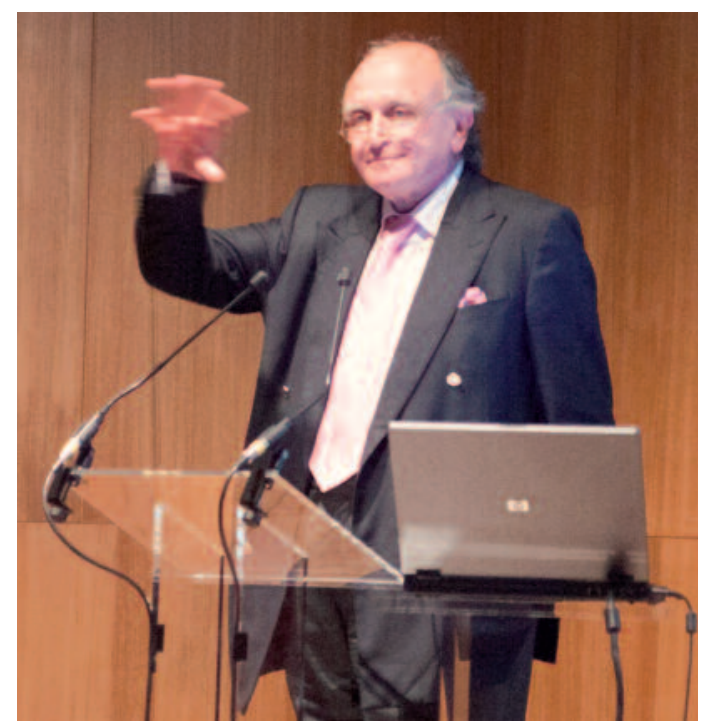

notamment en implantologie, et qu'elle délivre moins de rayonnement au patient qu'un scanner conventionnel. Elle est en outre insensible, contrairement à ce dernier, aux artéfacts causés par les obturations ou les prothèses métalliques présentes en bouches.

Le message délivré aux odonto-stomatologistes par le conférencier est simple, efficace, remarquablement illustré, et clair : "Toute douleur maxillaire évoluant depuis plusieurs mois est une lésion péri-apicale jusqu'à preuve cone beam haute résolution du contraire ".

\section{Syndrome du canal carpien Dr Jean-Pierre Ringuier, chirurgien-orthopédiste}

Décrit il y a près d'un siècle, ce syndrome se caractérise par des paresthésies, une douleur du poignet, une hypoesthésie, un déficit moteur dans les cas avancés et un problème à
I'opposition du pouce. À la longue, les muscles dans le territoire atteint peuvent s'hypotrophier. La radiographie et surtout l'électromyogramme sont contributifs. Sur le plan physio- 
pathologique, on distingue les ténosynovites non spécifiques des ténosynovites spécifiques. L'étiologie est essentiellement idiopathique bien que I'on retrouve un certain nombre de travailleurs manuels ou de patients traumatisés ou encore souffrant de polyartrite rhumatoïde. Notons que les odontostomatologistes ne constituent pas une population à risque

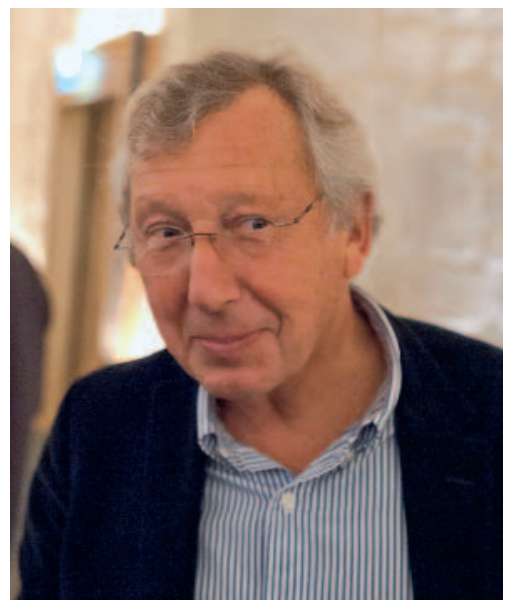
Le traitement est médical ou chirurgical avec des éléments à prendre en considération comme la durée de la compression nerveuse par rapport à l'âge du patient, ainsi que sa tolérance et les données chiffrées de l'électromyogramme. Le traitement chirurgical s'effectue par ouverture du ligament antérieur annulaire du carpe par techmême si la survenue d'un syndrome du canal nique à ciel ouvert ou technique endoscocarpien pose chez eux de gros problèmes dans leur travail quotidien. pique, la seconde méthode étant la plus usitée par le conférencier.

À en juger par le nombre de questions, d'ailleurs fort pertinentes, et la qualité des intervenants, cette conférence a tenu éveillé ses auditeurs jusqu'à une heure assez tardive où une collation bien méritée attendait tout le monde dans le cadre superbe de la Maison de la chasse et de la nature à l'Hôtel de Guénégaud. Nous vous espérons toujours aussi attentifs et nombreux à notre prochaine réunion. À l'année prochaine !

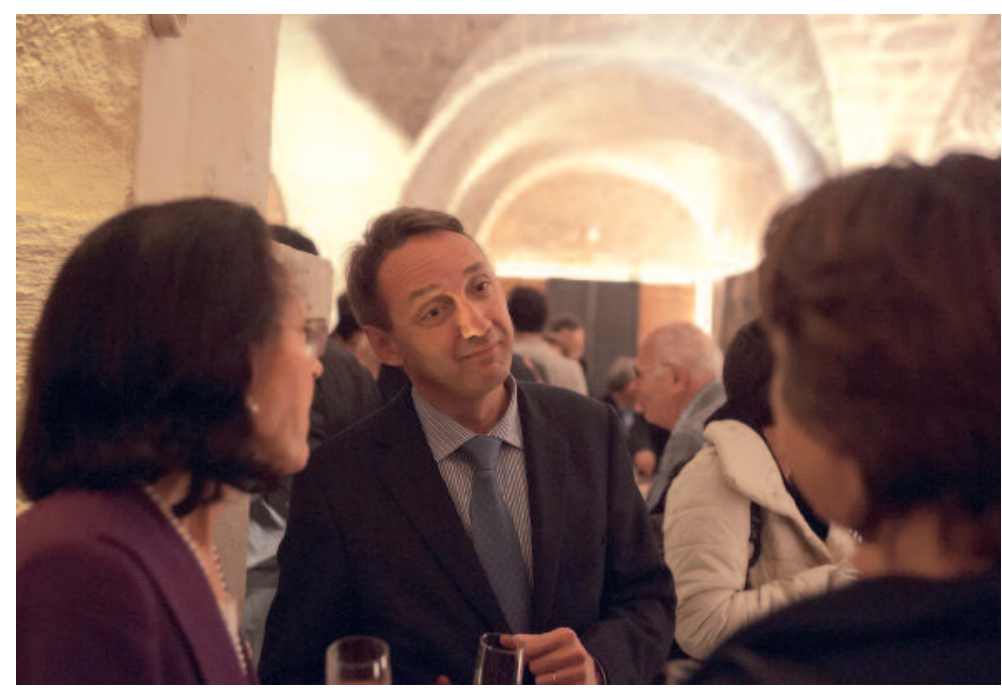

